\title{
Vortex nucleation barrier in superconductors beyond the Bean-Livingston approximation: A numerical approach for the sphaleron problem in a gauge theory
}

\author{
Andrea Benfenati $\odot,{ }^{*}$ Andrea Maiani $\odot,{ }^{\dagger}$ Filipp N. Rybakov $\odot$, and Egor Babaev \\ Department of Theoretical Physics, The Royal Institute of Technology, Stockholm SE-10691, Sweden \\ (Received 21 December 2019; revised manuscript received 19 March 2020; accepted 7 May 2020; \\ published 9 June 2020)
}

\begin{abstract}
The knowledge of vortex nucleation barriers is crucial for applications of superconductors, such as singlephoton detectors and superconductor-based qubits. Contrarily to the problem of finding energy minima and critical fields, there are no controllable methods to explore the energy landscape, identify saddle points, and compute associated barriers. Similar problems exist in high-energy physics where the saddle-point configurations are called sphalerons. Here, we present a generalization of the string method to gauge field theories, which allows the calculation of energy barriers in superconductors. We solve the problem of vortex nucleation, assessing the effects of the nonlinearity of the model, complicated geometry, surface roughness, and pinning.
\end{abstract}

DOI: 10.1103/PhysRevB.101.220505

In type-II superconductors, the Meissner state, characterized by the total magnetic field expulsion in the bulk, is stable up to the lower critical field $H_{\mathrm{c} 1}$. Above it, quantum vortices appear. However, the Meissner state can survive as a metastable state, causing the phenomenon known as magnetic superheating [1]. The presence of fluctuations can trigger the spontaneous decay of the metastable Meissner state through vortex formation. This phenomenon is the effect of a surface barrier, which hinders vortex nucleation from the sample boundaries [1,2]. The barrier disappears when the applied magnetic field exceeds a critical value, the nucleation field. In contrast to the potential barrier, the nucleation field is not only amenable to analytical treatment [3-11], but can be calculated with a controlled numerical accuracy from iterative simulations [12-14].

The full problem of the vortex entry barrier is not solved even for the simplest case of a semi-infinite superconductor with an ideal surface. The Bean-Livingston estimate [2] relies on the London model, but the result depends on the choice of cutoff, as emphasized by the authors themselves. The BeanLivingston approach has been extended to other geometries $[15,16]$. The effect of surface roughness is considerably more complicated. Only some approximate analytical approaches within the London model were advocated in Ref. [17]. However, roughness alters the core structure and therefore a

\footnotetext{
*alben@kth.se

$\dagger$ andrea.maiani@nbi.ku.dk
}

Published by the American Physical Society under the terms of the Creative Commons Attribution 4.0 International license. Further distribution of this work must maintain attribution to the author(s) and the published article's title, journal citation, and DOI. Funded by Bibsam. controllable method to solve the full nonlinear model is needed.

Fundamentally, the problem of a vortex entry barrier consists of finding the sphaleron, i.e., the saddle point which separates two stable states in a gauge theory [18-22]. In the case of vortex nucleation, it is an energy maximum of the minimum energy path between two states with different phase windings.

The knowledge of potential barriers for vortex nucleation is crucial for superconductor applications. The applications include current transmission, where the dissipationless state is lost if free vortices form, and superconductive rf cavities for particle accelerators where superconductive magnets often operate in a superheated state [11]. Recently, the problems of vortex entry barriers appeared in quantum technologies. In superconducting single-photon detectors, it is believed that the principle of operation consists in the creation of a currentcarrying state with a small potential barrier for vortex entry so that a single photon creates a vortex, hence a detectable signal $[23,24]$. However, small barriers yield spontaneous vortex nucleation caused by fluctuations, resulting in dark counts. The ability to calculate potential barriers would allow designing devices with significantly improved performances. Likewise, the knowledge of vortex entry barriers is crucial to design superconducting topological qubits [25-29], where the geometry of the device plays a central role. These devices often operate at temperatures near absolute zero. However, while a microscopically derived Ginzburg-Landau model applies only close to the critical temperature, many aspects of low-temperature vortex physics may under certain conditions be fittable by effective Ginzburg-Landau-type models [30]. In this Rapid Communication, we generalize to gauge theories the simplified string method [31]. This allows us to perform surface barrier calculations in type-II superconductors for vortex nucleation $\left(\Delta F_{\mathrm{n}}\right)$ and escape $\left(\Delta F_{\mathrm{e}}\right)$, by computing the minimum energy path of the transition in the Ginzburg-Landau 
theory. The results differ from the Bean-Livingston theory [2], which neglects the vortex-core and nonlinear effects. More importantly, the method allows taking into account the effect of surface roughness and the presence of pinning in the computation of the vortex nucleation barrier and the superheating field. The Ginzburg-Landau free-energy functional describing the superconductor in dimensionless units is

$$
\begin{aligned}
f[\mathbf{a}, \psi]= & \int_{\Omega} d \Omega\left(\frac{1}{2}\left|\left(-i \frac{2}{\kappa} \nabla+\mathbf{a}\right) \psi\right|^{2}\right. \\
& \left.+\frac{1}{2}\left(1-|\psi|^{2}\right)^{2}+\frac{(\nabla \times \mathbf{a}-\mathbf{h})^{2}}{2}\right) .
\end{aligned}
$$

The complex field $\psi=|\psi| e^{i \theta}$ describes the state of the superconductor. The vector potential $\mathbf{a}$ is related to the magnetic field by $\mathbf{b}=\nabla \times \mathbf{a}$. The coefficient $\kappa=\frac{\lambda}{\xi}$ is the GinzburgLandau parameter, i.e., the ratio of the magnetic field penetration depth $\lambda$ and the coherence length $\xi$ (we use the definition of coherence length with a factor $\sqrt{2}$ absorbed as in Ref. [32]). The external magnetic field $\mathbf{H}$ is expressed in units of the thermodynamic critical field $H_{\mathrm{c}}$, i.e., $h=H / H_{\mathrm{c}}$. The free energy $f=F / F_{0}$ is expressed in units of $F_{0}$, which in SI units is $F_{0}=\mu_{0} H_{\mathrm{c}}^{2} \lambda^{2} d$, where $d$ represents the thickness of the sample and $\mu_{0}$ is the vacuum permeability. Quantities in capital letters are intended in SI units, while variables in lowercase are dimensionless.

In a finite system, the Meissner state can survive in a metastable way for higher fields than $H_{\mathrm{cl}}$, i.e., up to the spontaneous nucleation field $H_{\mathrm{n}}$. In the absence of fluctuations, only when this field is exceeded, vortices nucleate from the boundaries. To introduce a vortex in the system, when $H<H_{\mathrm{n}}$, we need to overcome an energy barrier due to the surface. The calculation of this barrier is a nonlinear problem that is not amenable to analytical treatment. In this work, we generalize a numerical approach which was originally developed within the molecular dynamics field to study transitions between two metastable states through the identification of a minimum energy path [33]. The path is considered in the configuration space of the system, and it is parametrized by the transition coordinate $s \in[0,1]$. If one denotes by $\mathbf{q}(s)=[\mathbf{a}(s, \mathbf{r}), \psi(s, \mathbf{r})]$, the state of the system, then $\mathbf{q}(0)$ and $\mathbf{q}(1)$ are two equilibrium solutions corresponding to the minima of the Hamiltonian in Eq. (1). By varying $s$ from 0 to 1 , we observe the transition of the system from the initial state to the final state. One can assume that a potential force $\mathbf{g} \equiv-\nabla f=-\left(\frac{\delta f}{\delta \mathbf{a}}, \frac{\delta f}{\delta \psi^{*}}\right)$ acts on each point of the curve $\mathbf{q}(s)$. The minimum energy path is a trajectory in which the force g acting on each point is uniquely directed along the tangent vector $\partial \mathbf{q} / \partial s$, i.e.,

$$
\left|\nabla f \cdot \frac{\partial \mathbf{q}}{\partial s}\right|=\|\nabla f\|\left\|\frac{\partial \mathbf{q}}{\partial s}\right\|, \quad \forall s \in[0,1] .
$$

We emphasize that the optimal path defined by Eq. (2) does not correspond to the real time dynamics. It describes the most energetically favorable transformation undertaken by the system for the transition between the initial and the final state.

To identify the minimum energy path, we started from the well-known simplified string method [31] and developed a variant applicable to gauge field theories [34]. This algorithm evolves an initial guessed path in the configuration space, towards the minimal energy one. Consider a situation where we start from a Meissner state and end in the one-vortex state. To construct the initial guess, we used an ansatz for the single winded vortex state. In the initial state, for $s=0$, the vortex is outside of the domain and in the final state, for $s=1$, it lies in the origin. To each value of the transition coordinate $s$, there corresponds a particular configuration of the system. Hence, $s$ is not equivalent to the position of the center of the vortex because static vortex-core deformations correspond in general to different values of $s$. This method allows us to solve the full nonlinear problem in contrast to previous approaches, and obtain exact and quantitatively valid results. A previous study of the vortex entry barrier, based on the London model, was carried out by Bean-Livingston [2]. However, this introduced the uncontrollable approximation of considering the vortex core as a rigid cylinder of radius $\xi$, which neglects the physics of the core and the nonlinear effects.

The energy dependence $F(s)$ is a function with one or more maxima. At the summit of the potential barrier, the configuration of the system is a saddle point of the free-energy functional of Eq. (1), which in the context of gauge theories is called a sphaleron.

Once the minimum energy path is computed, we can define the nucleation barriers $\Delta F_{\mathrm{n}}=F_{\text {sphaleron }}-F_{\text {Meissner }}$ for a given magnitude of the external magnetic field $H$. Consequently, the nucleation field $H_{\mathrm{n}}$ is the external field needed to nullify the nucleation barrier, i.e., $\Delta F_{\mathrm{n}}\left(H_{\mathrm{n}}\right)=0$ [35]. Analogous definitions can be used for the escape barrier, i.e., the energy needed to expel one vortex from the superconductor. A quantitative description of nucleation and escape barriers is given in the Supplemental Material [34]. The minimum energy path contains more information than the height of the energy barrier as those paths are most likely to be followed in the nucleation process. This path helps us understand in detail how transitions between metastable states occur. In our framework, we can calculate the energy barrier free from any approximations, except fully controlled numerical errors. We begin by considering a vortex entry in a two-dimensional (2D) superconductor with flat surfaces. We find that the vortex always enters, following the minimum energy path, from the sides of the superconductor and never from the corners. Figure 1 shows the free-energy profile of the process. The barrier presents a single maximum corresponding to the sphaleron. The substantial core deformation confirms that this kind of problem is not in general treatable with controlled accuracy in the London limit.

For complex materials, the surface can have different roughness, doping, and oxidation, which strongly affects the vortex entry process. The knowledge of how impurities influence vortex physics is crucial for applications [36].

Let us consider randomly distributed inhomogeneities, with a decreasing density as we enter the sample, as shown in Fig. 2. To model inhomogeneities in a superconductor, we follow a procedure similar to the one outlined in Refs. [37-39], where we modify the quadratic term in Eq. (1) accordingly,

$$
\frac{1}{2}\left[1-|\psi(\mathbf{r})|^{2}\right]^{2} \rightarrow \frac{1}{2}\left[1+\sigma(\mathbf{r})-|\psi(\mathbf{r})|^{2}\right]^{2},
$$




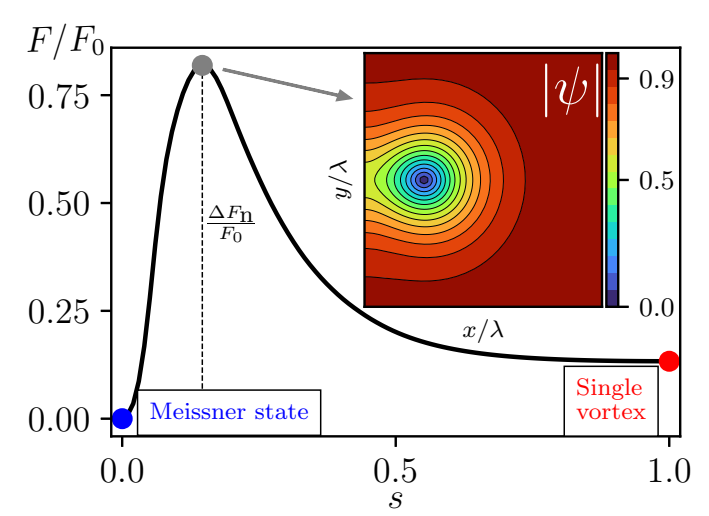

FIG. 1. The energy dependence and the saddle-point (sphaleron) configuration for the process of vortex entry from a straight edge in a two-dimensional superconductor. At the peak of the energy barrier, we have a substantial vortex-core deformation which cannot be described in the London limit, where $\psi$ is assumed to be constant. This shows how this kind of process, in general, cannot be treated in the London model, i.e., within the Bean-Livingston approach. The variable $s$ parametrizes the minimum energy path. In this example $H=0.6 H_{\mathrm{c}}$ and $\kappa=2$. The inset shows $|\psi|$ in the region when the nucleation takes place.

where $\sigma(\mathbf{r})$ is the inhomogeneity distribution. We tested different models for the inhomogeneity distribution with similar results. In the free-energy profile, depicted in Fig. 2, there is a global minimum in between the saddle points. This has the effect of increasing the nucleation barrier for a second vortex. In fact, in a clean sample, the entry barriers for the first and the second vortex are nearly the same. However, in the presence of inhomogeneities, we can calculate how the first vortex is pinned near the surface with the effect of increasing the nucleation barrier for the second one, as shown in Fig. 3.

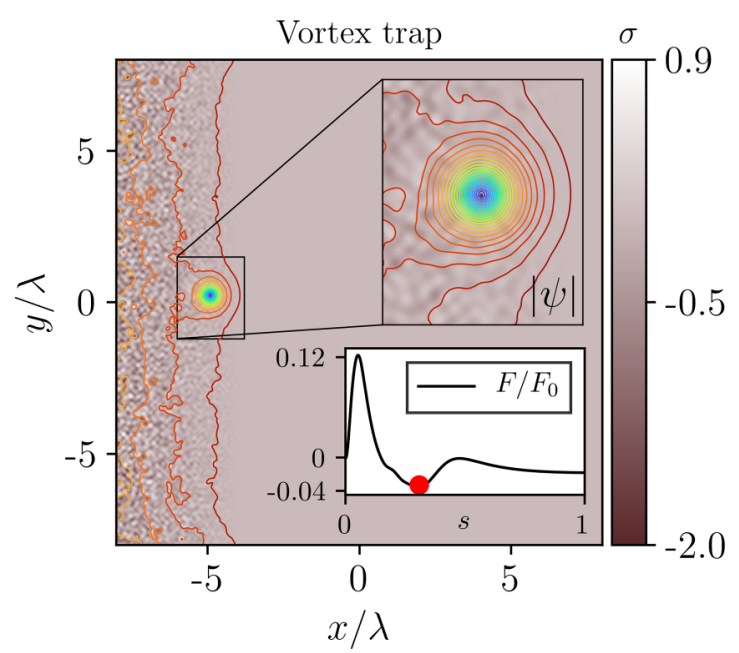

FIG. 2. Trapped vortex and corresponding energy profile for the minimum energy path in the case of a superconductor with inhomogeneities concentrated near the edge. The impurity layout $\sigma$ is normally distributed, and it is decreasing to zero as we proceed towards the bulk. On top of it, we plot isolines of the order parameter modulus $|\psi|$. For the color notation of isolines, see the color bar in Fig. 1. Moreover, we have $H=0.4 H_{\mathrm{c}}$ and $\kappa=5$.

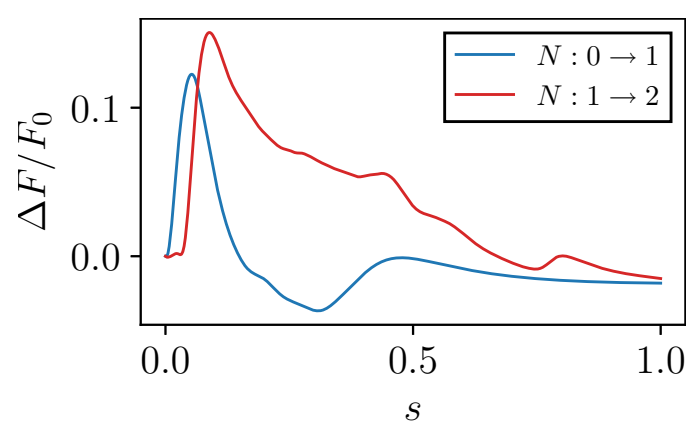

FIG. 3. Minimum free-energy paths for the first (blue) and second (red) vortex entry in the presence of the impurity modulation of Fig. 2. For the second entrance simulation, the initial condition was built by placing a vortex in the trap as shown in Fig. 2 (initial winding number $N=1$ ). Impurities pin the first vortex near the boundary, increasing the nucleation barrier of the second one.

Hence, with this method, it is possible to accurately predict and design impurity density profiles able to protect the sample from vortex entry by pinning vortices in an area near the surface.

Real samples in general have rough surfaces. It is empirically known that in the presence of rough surfaces, the vortex entry barrier is altered [1]. A rough surface represents a challenging problem for the calculation of both superheating fields [14] and barriers [17].

For a quasi-two-dimensional superconductor, we can describe a rough edge as a sequence of geometrical defects, forming dents in the sample, as sketched in Fig. 4. The method is also straightforwardly applicable to the three-dimensional case.

The vortex entry occurs from a single dent, where the barrier is suppressed the most. Therefore, it is relevant to study how the geometrical properties of one dent affect the nucleation barrier $\Delta F_{\mathrm{n}}$. As shown in Fig. 4(b), we can characterize the dent by its depth $l$ which can be comparable to or bigger than the characteristic length scales of the model (coherence length $\xi$ and penetration depth $\lambda$ ), and by an angle $\phi$ which determines its sharpness. Figure 5 compares the nucleation barriers $\Delta F_{\mathrm{n}} / F_{0}$ for different dent depths $l$, as a function of the sharpness angle $\phi$. For $\phi=\pi$ we have the limit of a perfectly straight edge. The green line shows the nucleation barrier for $l=\xi$. We notice that the energy barrier is unaltered by the presence of the dent with a comparable or smaller roughness. Hence, roughness profiles with depths of the order (a)
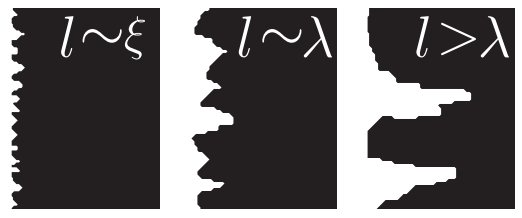

(b)

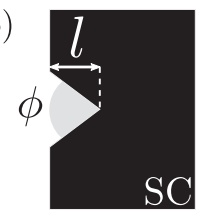

FIG. 4. (a) Examples of roughness profiles at the superconductor's edge tested in the simulations. In these three cases the profile is, going from left to right, comparable to the coherence length $\xi$, comparable to the penetration depth $\lambda$, and bigger than $\lambda$. (b) Model of a single dent: The depth is parametrized by $l$, while its sharpness by the angle $\phi$. The black color indicates the superconductor (SC). 


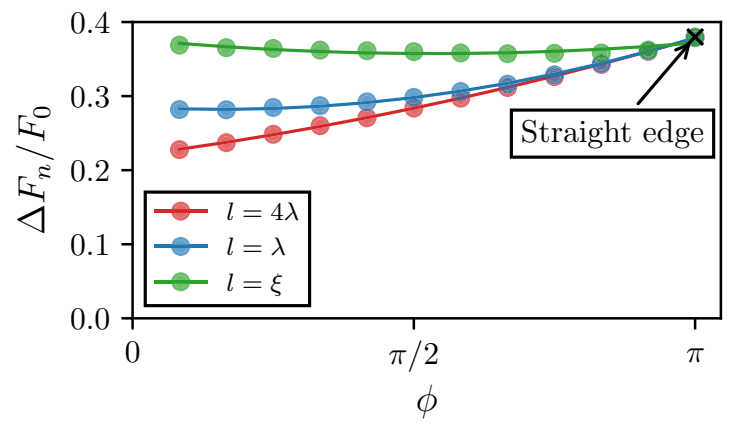

FIG. 5. Nucleation barrier computed for a dent with depth $l$ as a function of the angle $\phi$ as shown in Fig. 4(b). The system parameters are $\kappa=5$ and $H=0.4 H_{\mathrm{c}}$. The continuous lines are obtained by a quadratic regression (OLS) and agree with the input data. In particular, for the bigger dent, the nonlinear coefficient is negligible and the behavior is substantially linear.

of a coherence length have no effect on the nucleation barrier, independently of the sharpness of the dents. As $l$ increases, the barrier suppression becomes substantial, depending on the sharpness $\phi$. For $l=\lambda$ the dependence on $\phi$ is nonlinear, whereas for $l>\lambda$ (in our case $l=4 \lambda$ ), the barrier decreases linearly with $\phi$.

In conclusion, we formulated a method to compute the minimum energy path in a gauge theory. We applied this method to the problem of vortex nucleation in the GinzburgLandau model of superconductivity. We applied this method to solve the full nonlinear problem of vortex nucleation in the Ginzburg-Landau model of superconductivity. The method allows calculating vortex entry barriers in the presence of surface roughness and impurities. We showed that previously developed models to estimate the vortex entry barrier, based on the London approximation, are, in general, inadequate to describe the nucleation process even for a perfectly flat surface. We find that the surface roughness at the scale of coherence length affects the barriers insignificantly, and thus for superconductors with short-scale surface roughness, the main mechanism for the surface barrier reduction is the modulation of the superfluid density at larger length scales near the surface. The method straightforwardly applies to three-dimensional configurations and to the geometries where demagnetization fields are important [27]. Such vortex entry barriers are of key importance in the design of quantum devices such as single-photon detectors and qubits, as well as superconducting rf cavities, transmission lines, and magnets. Finally, the method is straightforwardly applicable to other gauge theories, different boundary conditions [46] which includes microscopic models of superconductivity, and models used in high-energy physics.

We thank Mats Barkman, Robert Vedin, and Oskar Palm for useful comments. We gratefully acknowledge the support of NVIDIA Corporation with the donation of the Quadro P6000 GPU used for this research. Published with support from the Längman Culture Foundation. The work was supported by the Swedish Research Council Grants No. 6422013-7837, No. 2016-06122, and No. 2018-03659, and Göran Gustafsson Foundation for Research in Natural Sciences and Medicine and Olle Engkvists Stiftelse.

A.M. and A.B. contributed equally to this work.
[1] V. V. Schmidt, The Physics of Superconductors: Introduction to Fundamentals and Applications (Springer, Berlin, 2013).

[2] C. P. Bean and J. D. Livingston, Phys. Rev. Lett. 12, 14 (1964).

[3] P. De Gennes, Solid State Commun. 3, 127 (1965).

[4] L. Kramer, Phys. Rev. 170, 475 (1968).

[5] H. J. Fink and A. G. Presson, Phys. Rev. 182, 498 (1969).

[6] P. V. Christiansen, Solid State Commun. 7, 727 (1969).

[7] L. Kramer, Z. Phys. A 259, 333 (1973).

[8] A. J. Dolgert, S. J. Di Bartolo, and A. T. Dorsey, Phys. Rev. B 53, 5650 (1996).

[9] G. Catelani and J. P. Sethna, Phys. Rev. B 78, 224509 (2008).

[10] M. K. Transtrum, G. Catelani, and J. P. Sethna, Phys. Rev. B 83, 094505 (2011).

[11] D. B. Liarte, S. Posen, M. K. Transtrum, G. Catelani, M. Liepe, and J. P. Sethna, Supercond. Sci. Technol. 30, 033002 (2017).

[12] C. Bolech, G. C. Buscaglia, and A. López, Phys. Rev. B 52, R15719 (1995).

[13] S. Kim, J. Burkardt, M. Gunzburger, J. Peterson, and C.-R. Hu, Phys. Rev. B 76, 024509 (2007).

[14] A. R. Pack, J. Carlson, S. Wadsworth, and M. K. Transtrum, Phys. Rev. B 101, 144504 (2020)

[15] V. P. Galaiko, Zh. Eksp. Teor. Fiz. 50, 1322 (1966) [Sov. Phys. JETP 23, 878 (1966)].

[16] A. L. Fetter, Phys. Rev. B 22, 1200 (1980).
[17] F. Bass, V. Freilikher, B. Y. Shapiro, and M. Shvartser, Physica C: Superconductivity 260, 231 (1996).

[18] V. Rubakov, Classical Theory of Gauge Fields (Princeton University Press, Princeton, NJ, 2009).

[19] N. Manton and P. Sutcliffe, Topological Solitons (Cambridge University Press, Cambridge, UK, 2004).

[20] H. Năstase, Classical Field Theory (Cambridge University Press, Cambridge, UK, 2019).

[21] E. W. Kolb and M. S. Turner, The Early Universe (Frontiers in Physics) (Westview Press, Cambridge, MA, 1994).

[22] M. Shifman, Advanced Topics in Quantum Field Theory: A Lecture Course (Cambridge University Press, Cambridge, 2012).

[23] C. M. Natarajan, M. G. Tanner, and R. H. Hadfield, Supercond. Sci. Technol. 25, 063001 (2012).

[24] I. Esmaeil Zadeh, J. W. N. Los, R. B. M. Gourgues, V. Steinmetz, G. Bulgarini, S. M. Dobrovolskiy, V. Zwiller, and S. N. Dorenbos, APL Photon. 2, 111301 (2017).

[25] A. Y. Kitaev, Phys. Usp. 44, 131 (2001).

[26] C. Nayak, S. H. Simon, A. Stern, M. Freedman, and S. Das Sarma, Rev. Mod. Phys. 80, 1083 (2008).

[27] J. D. S. Bommer, H. Zhang, O. Gül, B. Nijholt, M. Wimmer, F. N. Rybakov, J. Garaud, D. Rodic, E. Babaev, M. Troyer, D. Car, S. R. Plissard, E. P. A. M. Bakkers, K. Watanabe, T. Taniguchi, and L. P. Kouwenhoven, Phys. Rev. Lett. 122, 187702 (2019). 
[28] R. M. Lutchyn, J. D. Sau, and S. Das Sarma, Phys. Rev. Lett. 105, 077001 (2010).

[29] R. M. Lutchyn, G. W. Winkler, B. van Heck, T. Karzig, K. Flensberg, L. I. Glazman, and C. Nayak, Science 367, eaav3392 (2020).

[30] M. Silaev and E. Babaev, Phys. Rev. B 85, 134514 (2012).

[31] Weinan E, W. Ren, and E. Vanden-Eijnden, J. Chem. Phys. 126, 164103 (2007).

[32] B. V. Svistunov, E. S. Babaev, and N. V. Prokof'ev, in Superfluid States of Matter (CRC Press, Boca Raton, FL, 2015), pp. 1-546.

[33] Weinan E and E. Vanden-Eijnden, Annu. Rev. Phys. Chem. 61, 391 (2010).

[34] See Supplemental Material at http://link.aps.org/supplemental/ 10.1103/PhysRevB.101.220505 for details of the gauged string method developed for this problem. The Supplemental Material includes Refs. [40-45].

[35] Note that the nucleation field $H_{\mathrm{n}}$ is defined as the external field needed for suppressing the barrier and causing a single spontaneous vortex entry. The superheating field $H_{\mathrm{sh}}$, often referred to in the literature, is defined as the upper metastability limit of the Meissner phase for large and homogeneous superconductors.

[36] K. Fossheim and A. Sudboe, Superconductivity: Physics and Applications (Wiley, New York, 2004).

[37] S. J. Chapman, Q. Du, and M. D. Gunzburger, Eur. J. Appl. Math. 6, 97 (1995).

[38] Q. Du, M. D. Gunzburger, and J. S. Peterson, Phys. Rev. B 51, 16194 (1995).

[39] J. Deang, Q. Du, M. Gunzburger, and J. Peterson, Philos. Trans. R. Soc. London, Ser. A 355, 1957 (1997).

[40] P. F. Bessarab, V. M. Uzdin, and H. Jónsson, Comput. Phys. Commun. 196, 335 (2015).

[41] G. Henkelman, B. P. Uberuaga, and H. Jńsson, J. Chem. Phys. 113, 9901 (2000).

[42] E. F. Koslover and D. J. Wales, J. Chem. Phys. 127, 134102 (2007).

[43] D. Sheppard, R. Terrell, and G. Henkelman, J. Chem. Phys. 128, 134106 (2008).

[44] H. A. Kramers, Physica 7, 284 (1940).

[45] J. S. Langer, Ann. Phys. 54, 258 (1969).

[46] A. Samoilenka and E. Babaev, Phys. Rev. B 101, 134512 (2020). 